\title{
Detection of Bio-film Production among the Most Frequent Bacterial Isolates in Cases of Chronic Suppurative Otitis Media: A Cross-sectional Study
}

\author{
Priya Bhat ${ }^{1}$, Sameer Peer ${ }^{2 *}$ and Raksha Yoganand ${ }^{1}$ \\ ${ }^{1}$ Department of Microbiology, Employees State Insurance Corporation Medical College and \\ Hospital, Rajajinagar, Bengaluru - 560010, Karnataka, India \\ ${ }^{2}$ Department of Neuroimaging and interventional Radiology, National Institute of Mental \\ Health and Neurosciences, Bengaluru-560029, Karnataka, India \\ *Corresponding author
}

\section{A B S T R A C T}

\section{Keywords}

Biofilm, Antibiotic, Chronic

Suppurative Otitis

Media,

Tympanoplasty

Article Info

Accepted:

18 May 2020

Available Online:

10 June 2020
To detect biofilm production among the most common micro-organisms isolated from CSOM cases. Five hundred consecutive patients with a diagnosis of CSOM were enrolled in the study. Organisms were isolated using standard culture methods and biochemical reactions. Antibiotic sensitivity pattern was determined according to the Clinical and Laboratory Standards Institute (CLSI) guidelines. Biofilm production was detected using Tube method and microtitre plate method. The most common organism isolated was Pseudomonas aeruginosa in 220 cases(44\%) followed by Staphylococcus aureus in 110 cases (22\%). Out of 186 isolates tested for biofilm production, 149 isolates (80\%) showed biofilm production. Out of the moderately positive and strongly positive biofilm producers, $90 \%$ isolates were from unsafe CSOM. High proportion of isolates from CSOM cases shows biofilm production. Biofilm production may account for antibiotic resistance, recurrence of CSOM and failure of surgical procedures in CSOM cases.

\section{Introduction}

Chronic suppurative otitis media (CSOM) is defined as chronic inflammation of middle ear and mastoid cavity that presents with recurrent ear discharge of more than three months duration through a perforated tympanic membrane (WHO, 2004).CSOM is a major health problem in developing countries causing serious local damage and life threatening complications. It is an important cause of preventable hearing loss in developing countries. The infection may begin in childhood as a complication of untreated or inadequately treated Acute Suppurative Otitis Media (ASOM) or may be chronic in onset. The bacteria may gain entry to the middle ear through a chronic perforation (Afolabi et al., 2012). The spread of micro-organisms to the adjacent structures 
of the ear may cause local damage or intracranial and extra cranial complications, ranging from persistent otorrhoea, mastoiditis, labyrinthitis, facial nerve palsy, meningitis, intracranial abscess or thrombosis and sepsis (Kumar et al, 2015). The most common microorganisms found in isolates from ear discharge in CSOM are Pseudomonas aeruginosa, Staphylococcus aureus, Klebsiella pneumoniae, Proteus mirabilis, Escherichia coli, Aspergillus spp., Candida spp. and these vary in different geographical distributions (Yeo et al., 2007).Biofilms are adherent communities of bacteria contained within a complex matrix.

Host immune responses to planktonic species have been relatively well characterized and also how they modulate anti-bacterial effector mechanisms when organized in this protective milieu (Hank et al., 2012).Biofilms act as non selective physical barriers that obstruct antibiotic diffusion and hinder the cellular and humoral immune responses (Marchant et al., 2013). They have a characteristic physiology and architecture that form the basis of biofilm resistance to many antibiotics and mechanisms of host defense (Otto, 2013).

Bacteria may communicate with each other through diffusible molecules within the milieu of a biofilm through a mechanism known as quorum sensing ( $\mathrm{Li}$ and Tian, 2012). The purpose of this study was to determine the prevelance of biofilm producing bacteria among the most common bacterial isolates in cases of CSOM.

An epidemiological knowledge of the prevalence of biofilm production in CSOM is necessary as biofilm production may account for recurrence of CSOM after treatment, failure of surgery and resistance to antibiotic therapy. Also, we may be able to institute such therapies which may inhibit biofilm production (Rabin et al., 2015).

\section{Materials and Methods}

This prospective study was conducted in the Department of Microbiology in association with Department of Otorhinolaryngology in a tertiary care university hospital in Bangalore, India.

\section{Study period}

The study was conducted for a period of 18 months from January 2017 to June 2018.

\section{Study population}

500 patients with clinical diagnosis of CSOM attending OPD and IPD of ENT department of our institute who satisfied the inclusion criteria were enrolled for the study.

\section{Ethical consideration}

Approval was obtained from the Institutional ethics committee before the commencement of the study. Informed consent was obtained from all the patients who participated in the study.

\section{Statistical analysis}

Data was entered on Microsoft Excel 2016 for analysis. Data was represented in form of numbers and percentages. Analysis was done using descriptive statistics.

\section{Sample size estimation}

All ear discharge samples from cases of CSOM with a minimum of 500 consecutive non-duplicate isolates will be included. Sample size has been calculated to be 500 depending upon previous years statistics.

\section{Inclusion criteria}

Patients diagnosed as CSOM after thorough 
clinical evaluation i.e. the patients having ear discharge of more than 3 months.

Patients who were not on antibiotic (both systemic and topical) treatment for minimum of 24 hours prior to sample collection.

\section{Exclusion criteria}

Patients suffering from CSOM who are on systemic antibiotics in the past 7 days of presentation.

Patients who are on topical medications to the ear.

Patients having ear discharge due to some traumatic or neoplastic condition.

Patients having ear discharge arising from otitis externa.

\section{Sample collection}

The ear discharge was collected using sterile swabs under aseptic precautions with the aid of an aural speculum, prior to the instillation of any topical medication. Using standard sterile technique, two swabs were taken.

Specimen processing

\section{Direct gram stain}

Shows possible pathogens present in sample

\section{KOH mount}

Detection of fungal elements.

\section{Interpretation of bacterial culture}

The swab on reaching the laboratory was inoculated on the following culture media

5\% Sheep Blood agar plate

MacConkey agar plate

Thioglycollate broth
After overnight incubation at $37^{\circ} \mathrm{C}$ aerobically, the plates were examined for growth and culture characteristics were identified. The isolates were identified by Grams stain morphology, motility, culture characteristics and biochemical reactions by the standard techniques. The isolated colonies depending on the Gram reaction were subjected to following biochemical tests for identification:

If gram positive cocci were found, then catalase test was done. For catalase positive isolates, tube coagulase was done -Tube coagulase positive were identified as Staphylococcus aureus whereas tube coagulase negative were Coagulase negative staphylococcus aureus (CONS). The identification of Staphylococcus aureus is shown in Figure 1.

If Gram negative bacilli were seen, the colonies were subjected to the following tests and biochemical reactions using standard microbiological techniques.

Catalase test

Oxidase test

Nitrate reduction test

Hugh-Leifson's Oxidation Fermentation test Indole test

Methyl red test

Voges Proskauer test

Simmon's Citrate utilization test

Christensen's Urease test

Mannitol motility

Triple sugar iron agar

1\% Sugar fermentation tests Glucose, Sucrose, Maltose, Mannitol.

Lysine decarboxylase, Ornithine decarboxylase and Arginine dihydrolase test. Isolates that were Gram Negative bacilli, catalase positive, oxidase positive and motile by hanging drop were identified as Pseudomonas aeruginosa. 
The culture characteristics of Pseudomonas aeruginosa are shown in Figure 2.

\section{Antibiotic susceptibility}

The isolates were subjected for antibiotic susceptibility testing by employing KirbyBauer standard disc diffusion method on Muller- Hinton agar according to the Clinical and Laboratory Standards Institute (CLSI) guidelines (M100-S24).

\section{Biofilm production}

Biofilm production among Pseudomonas aeruginosa and Staphylococcus aureus isolates was tested by following methods:

\section{Tube method}

Tubes and incubated at $37{ }^{0} \mathrm{C}$ for $48 \mathrm{hrs}$. Content decanted and tubes washed with Normal saline and left for air dry, then stained with crystal violet $(0.1 \% \mathrm{w} / \mathrm{v})$ and rotated to ensure uniform staining, again washed with distilled $\mathrm{H}_{2} \mathrm{O}$ and dried in inverted position.

\section{Scoring of biofilm production}

The biofilm production was scored in a qualitative manner comparing the test sample with positive and negative controls as depicted in figure 3. The grading was done as: 0-absent,1-weakly positive,2-moderately positive,3-strongly positive.

\section{Microtitre plate method}

Pseudomonas aeruginosa and Staphylococcus aureus cultures were inoculated in $3-5 \mathrm{ml}$ BHI broth and incubated for $24 \mathrm{hrs}$ at 37 ${ }^{0} \mathrm{C}$,cultures diluted 1:100 in BHI and $100 \mathrm{ml}$ of each diluted culture was pipetted in each well of 96 well flat bottom microtiter plate, covered and incubated at $37{ }^{0} \mathrm{C}$ for $48 \mathrm{hrs}$., washed with Normal saline, $125 \mathrm{ml}$ of $0.1 \%$ crystal violet(w/v) solution put to each well, stain removed, plate washed with distilled water, left for air dry. Optical Density (OD) of each well was measured at $630 \mathrm{~nm}$ and performed in duplicate.

Characterization of isolates based on biofilm production was done based on cut-off OD (OD ${ }_{\text {CUT }}$ ) measured as:

$\mathrm{OD}_{\mathrm{CUT}}=\mathrm{OD}_{\mathrm{avg}}$ of negative control+3(SD) of OD of negative control).

Where $\mathrm{OD}_{\mathrm{avg}}-$ Average Optical Density, SD - Standard Deviation

The interpretation of biofilm production using microtitre plate method is shown as in Figure 4 and Figure 5.

\section{Results and Discussion}

500 patients were included in the study. $63 \%$ of the patients were males $(n=315)$ while $37 \%$ of patients were females $(n=185)$. Highest incidence of CSOM was found to be among patients in the age group of 20-29 years $(32.8 \%)$ while the lowest incidence was found in patients $\geq 60$ years age $(3.2 \%)$. The most common presenting complaint was ear discharge for more than 3 months (93\%), followed by decreased hearing (66\%). Upper respiratory tract infection was noted in $30 \%$ cases and allergic history was noted in $12 \%$ cases. $25 \%$ cases had a past history of modified radical mastoidectomy with tympanoplasty (Type 2). Tonsillitis and Deviated nasal septum was noted in 5\% and $3 \%$ respectively. Tuberculosis and adenoids were noted in $2 \%$ cases. Stroke( $1 \%)$, MillerFischer syndrome(1.6\%), Extrapulmonary tuberculosis $(0.4 \%)$ and nasal carcinoma $(0.4 \%)$ were the other co-morbidities among the patients. The most common organism isolated was Pseudomonas aeruginosain 220 cases $(44 \%)$ followed by Staphylococcus 
aureusin 110 cases (22\%). Methicillin resistant Staphylococcus aureus(MRSA) was isolated in 15 cases. CONS were isolated in 40 cases $(8 \%)$. Fungi were isolated in $6 \%$ cases. In mixed culture growth, the most common isolates were Pseudomonas aeruginosa and Klebsiella pneumoniae (2.8\%) followed by Pseudomonas aeruginosa and Escherichia coli (2.6\%).

Attico-antral type of CSOM was found in 150 cases $(30 \%)$ while 350 cases $(70 \%)$ were diagnosed with tubo-tympanic type of CSOM. Among the cases with attico-antral CSOM(Unsafe type), the most common organism isolated was Pseudomonas aeruginosa (54.6\%) followed by Staphylococcus aureus(32\%) and Klebsiella species $(6.6 \%)$.

Among Pseudomonas aeruginosa isolates, highest sensitivity was noted for Piperacillintazobactam (97\%), Meropenem (95\%), Cefoperazone (93\%) and Piperacillin (93\%). Only $68 \%$ isolates of P.aeruginosa were found to be sensitive to Ciprofloxacin.

$100 \%$ of Staphylococcus aureus isolates were found to be sensitive to Linezolid and Teicoplanin. $87 \%$ of S.aureus isolates were Cefoxitin sensitive (MSSA). 15\% of S.aureus isolates were found to be Cefoxitin resistant (MRSA). Highest resistance among S.aureus isolates was found for Penicillin(81\%) followed by Ciprofloxacin (65\%), Ampicillin(63\%) and AmoxicillinClavulanate $(54 \%)$.

\section{Biofilm production in Pseudomonas aeruginosa and Staphylococcus aureus}

Out of 186 isolates tested for biofilm production, 149 isolates $(80 \%)$ showed biofilm production. $50 \%$ of biofilm producing isolates were moderately positive, $47 \%$ were weakly positive while $3 \%$ were strongly positive. Out of the moderately positive and strongly positive biofilm producers $(\mathrm{n}=79)$, $90 \%$ isolates were from unsafe CSOM $(n=71)$ out of which $57(80 \%)$ were Pseudomonas aeruginosa while $14 \quad(20 \%)$ were Staphylococcus aureus. $86 \%$ isolates $(\mathrm{n}=12)$ of Staphylococcus aureus were MRSA while $14 \%$ were $\operatorname{MSSA}(\mathrm{n}=2)$.

All these patients with unsafe CSOM had a history of recurrent CSOM and were on antibiotic therapy for many years ( mean time on antibiotic treatment -8 years ). 60 patients $(84.5 \%)$ with unsafe CSOM with detection of moderately positive or strongly positive biofilm production had underwent modified radical mastoidectomy (MRM) with type 2 tympanoplasty in the past. The results of biofilm production are summarized in figure 6.

Chronic Suppurative Otitis Media(CSOM) is considered as a major public health problem in the developing world and India is one of the countries with high prevalence (WHO, 2004).It is a persistent disease with risk of irreversible complications and is an important cause of preventable hearing loss in adults and children (Berman, 1995).

Analysis of bacterial profile of the present study showed that Gram negative bacilli outnumbered Gram positive cocci among the isolates from the cases of CSOM which is similar to previous studies (Naheed et al., 2003 and Kulchal et al., 2010) The predominant pathogen was Pseudomonas aeruginosa (44\%) followed by Staphylococcus aureus (22\%), Acenitobacter (4\%), Klebsiella pneumoniae (6\%), Escherichia coli(4\%), and Proteus mirabilis(3\%). Similar findings have been noted in previous studies by (Shyamala et al., 2012).

The microbial profile may vary between 
different regions based on patient population and geographical distribution and hence necessitates the need for frequent analysis and update of the microbial profile in every region.

Antibiotic susceptibility pattern of P.aeruginosa revealed $100 \%$ susceptibility to Colistin, while $97 \%$ of the isolates were sensitive to Piperacillin-tazobactum, 95\% were sensitive to Meropenem, 93\% isolates were sensitive to Piperacillin, $93 \%$ to Cefoperazone, $90 \%$ to Imipenem, $81 \%$ to Amikacin, $75 \%$ to Gentamicin and $68 \%$ to Ciprofloxacin. Pseudomonas aeruginosa isolates were found to be more sensitive to Amikacin than Gentamicin and this finding is similar to previous studies in Sharma et $\mathrm{al}^{16}$ who reported $83.9 \%$ sensitivity to Amikacin and $70.5 \%$ sensitivity to Gentamycin.

Similar findings were documented in previous studies(Saini et al., 2005 and Wario et al., 2006). Fluoroquinolones inhibits the bacterial DNA gyrase or the topoisomerase II thereby inhibiting DNA transcription and replication. They have a broad range of activity and found to be active against Pseudomonas aeruginosa.

In the present study $52 \%$ of Gram negative isolates and $68 \%$ of P.aeruginosa were found to be sensitive to ciprofloxacin which is similar to the sensitivity reported in previous studies (Kumar R et al., 2015 and Kumar S et al., 2012).

$100 \%$ isolates of Staphylococcus aureus were sensitive to Linezolid and Teichoplanin. 65\% of Staphylococcus aureus isolates were resistant to Ciprofloxacin and 54\% were resistant to Amoxicillin-Clavulanate. This indicates high level of resistance among Staphylococcus aureus for $\beta$-Lactams and Fluoroquinolones. In a previous study, out of 181 isolates, $48 \%$ of isolates were Staphylococcus aureus and all were found to be methicillin sensitive (Prakash et al., 2013). Biofilm production by bacteria has been implicated in the pathogenesis of many chronic infections including CSOM.

Biofilm production is considered as one of the mechanisms which may contribute to the bacterial resistance to antibiotics and other antimicrobial compounds (Stewart and Costerton, 2001). Biofilms are difficult to eradicate and may cause recurrent infections (Donelli and Vuotto, 2014).Biofilms attach firmly to damaged tissue, such as exposed osteitic bone and ulcerated middle ear mucosa, or to otological implants such as tympanostomy tubes (Wang et al., 2014).

The critical first event in formation of biofim is adherence to host tissue or to foreign body surfaces. During insertion of a device such as a tympanostomy tube, there may be inoculation of bacteria from the skin and mucous membranes of the host tissue. An amorphous extracellular material composed of bacterial products, such as teichoic acids, proteins, polysaccharides, and extracellular DNA (eDNA) and host products, encases the bacterial agglomerates within a biofilm (Schommer et al., 2011).

Biofilms may be formed on the abiotic surfaces of medical devices or on biotic surfaces, such as host tissue (Becker et al., 2014). The development of a biofilm requires a combination of adhesive forces and disruptive forces. Adhesive forces are required for the colonization of surfaces and for cell-cell interaction.

Disruptive forces are needed for nutrient delivery to all biofilm cells through fluid filled channels and also for the detachment of cell clusters from the biofilm, which facilitates the dissemination of infection (Otto, 2013). 
Biofilm is formed as a result of various steps which are summarized below:

Attachment of bacteria to biotic or abiotic surface.

Multiplication and accumulation in multilayered cell aggregates.

Growth and maturation of the the biofilm into a thick, structured layer.

Dissociation and disseminate via the bloodstream.

To mediate attachment to abiotic surfaces or host tissues, proteinaceous and nonproteinaceous adhesins are required. Proteinaceous adhesins of bacteria have been classified into covalently surface-anchored proteins, also called cell wall anchored (CWA) proteins and noncovalently surfaceassociated proteins which include the autolysin/adhesin family, and the membrane spanning proteins (Becker et al., 2014).

Among the non-proteinaceous adhesins are the polysaccharide intercellular adhesin (PIA) (also known as poly-N-acetylglucosamine [PNAG]) as well as wall teichoic and lipoteichoic acids (Becker et al., 2014). Within a mature biofilm, multiple fluid-filled channels are formed which ensure the delivery of oxygen and nutrients to the bacterial cells located within the deeper layers of the biofilm (Costerton et al., 1999).

After adhesion, the bacteria proliferate and accumulate intomultilayered cell clusters. This process is mediated by the polysaccharide intercellular adhesin (PIA). The genes which are responsible for PIA formation are located on the ICA (intercellular adhesion) operon.

The ICA operon consists of two genes i.e.icaR (regulating) gene and icaABCD (biosynthetic) genes (Longauerova, 2006). Upon biofilm maturationa variety of extracellular enzymatic activities or phenol soluble modulins (PSMs) may cause dissociation of bacteria or bacterial clusters and may lead to dissemination of the infection into the blood stream (Becker et al., 2014).

In our study biofilm production was seen in $30 \%$ of unsafe CSOM cases. In a study, biofilm production was noted in $75 \%$ cases of unsafe CSOM and was significantly more common in unsafe CSOM than safe CSOM (Aronto et al., 2015). In another study, biofilms were present in $60 \%$ patients with CSOM as compared with $10 \%$ of controls who had ear surgeries for other causes (Lee et al., 2009).

The present study evaluated 186 isolates of Pseudomonas aeruginosa and Staphylococcus aureus for biofilm production. We found out of 186 isolates tested for biofilm production, 149 isolates (80\%) showed biofilm production. $50 \%$ of biofilm producing isolates were moderately positive, $47 \%$ were weakly positive while $3 \%$ were strongly positive.

Out of the moderately positive and strongly positive biofilm producers, $90 \%$ isolates were from unsafe CSOM out of which 57(80\%) were Pseudomonas aeruginosa while 14 (20\%) were Staphylococcus aureus.

$86 \%$ isolates of Staphylococcus aureus isolated from unsafe CSOM showed Methicillin resistance and these patients had a past history of MRM with Type 2 tympanoplasty, presented with recurrences and were on antibiotic treatment since many years.

Biofilm formation may explain the etiopathogenesis of recurrence and resistance pattern in unsafe CSOM. Determining the type of bacteria that form biofilm in unsafe CSOM will help in better management in recurrent CSOM cases. 


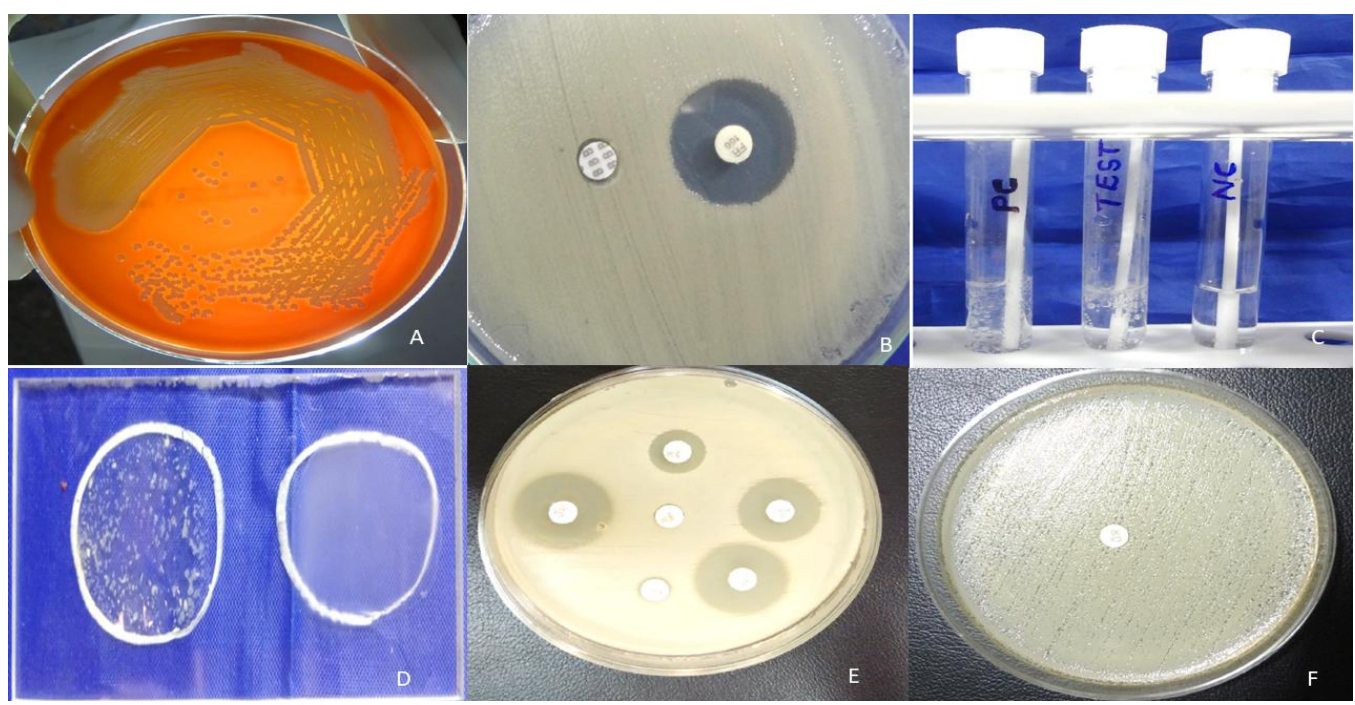

Figure.1 Characteristic features of Staphylococcus aureus. (A) Colonies of Staphylococcus aureus on blood agar. (B) Susceptibility to bacitracin and Furazolidone is seen in Staphylococcus aureus. (C) Staphylococcus aureus shows positive catalase test. (D) Staphylococcus aureus shows positive slide coagulase test. (E) Antibiotic susceptibility test using Kirby-Bauer standard disc diffusion method on Muller- Hinton agar according to the Clinical and Laboratory Standards Institute (CLSI) guidelines (M100-S24). Methicillin sensitive Staphylococcus aureus (MSSA) is noted. (F) Antibiotic susceptibility test using Kirby-Bauer standard disc diffusion method on Muller- Hinton agar according to the Clinical and Laboratory Standards Institute (CLSI) guidelines (M100-S24). Methicillin resistant Staphylococcus aureus (MRSA) is noted

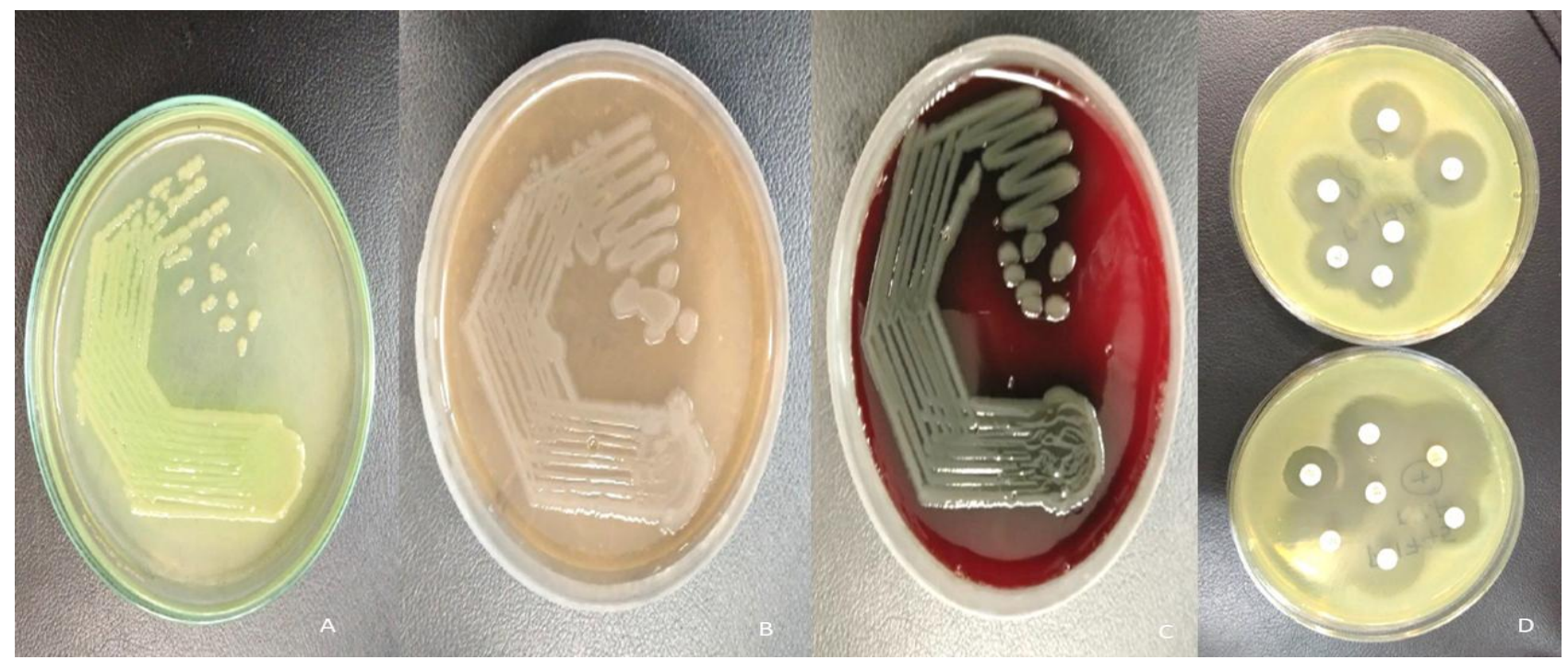

Figure.2 Culture characteristics of Pseudomonas aeruginosa. Colonies of Pseudomonas aeruginosa on Nutrient agar (A), MacConkey agar(B) and Blood agar(C). (D) Antibiotic susceptibility test using Kirby-Bauer standard disc diffusion method on Muller- Hinton agar according to the Clinical and Laboratory Standards Institute (CLSI) guidelines (M100-S24). Among Pseudomonas aeruginosa isolates, highest sensitivity was noted for Piperacillintazobactam (97\%), Meropenem (95\%), Cefoperazone (93\%) and Piperacillin (93\%). Only 68\% isolates of P.aeruginosa were found to be sensitive to Ciprofloxacin 


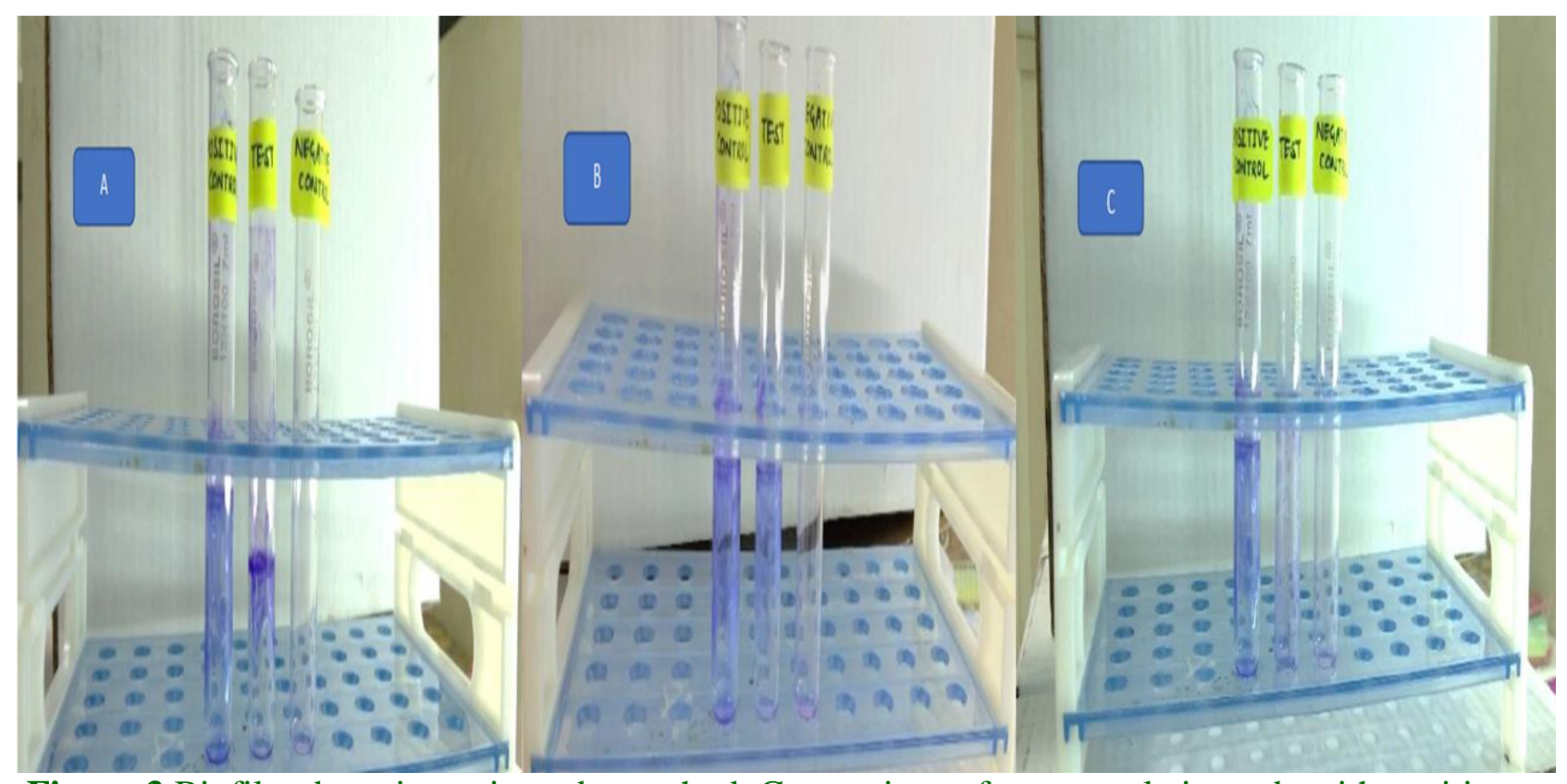

Figure.3 Biofilm detection using tube method. Comparison of test sample is made with positive and negative controls. (A) Strongly positive biofilm production. (B) Moderately positive biofilm production. (C) Weakly positive biofilm production
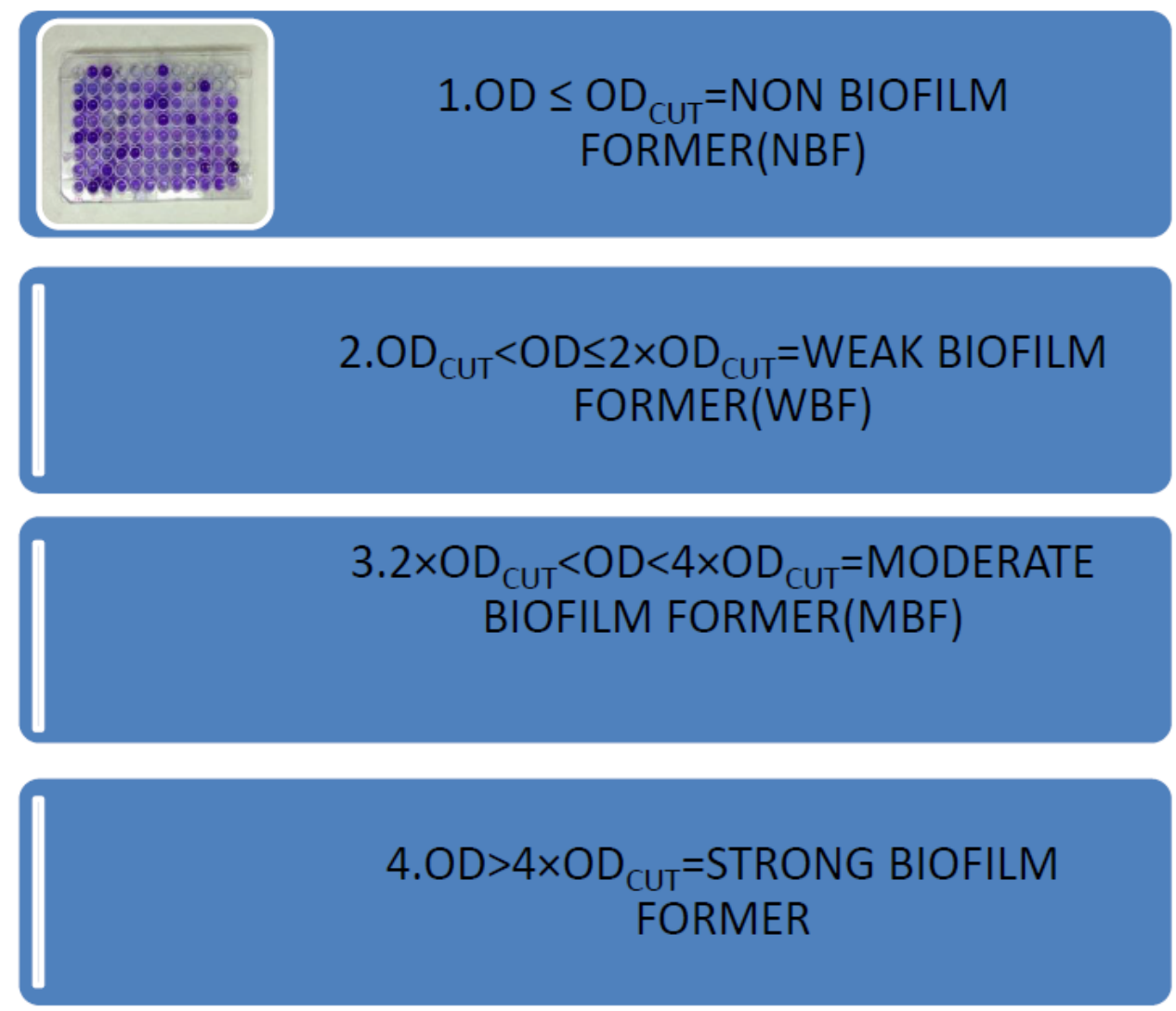

Figure.4 Interpretation of biofilm production using micro-titre plate method 


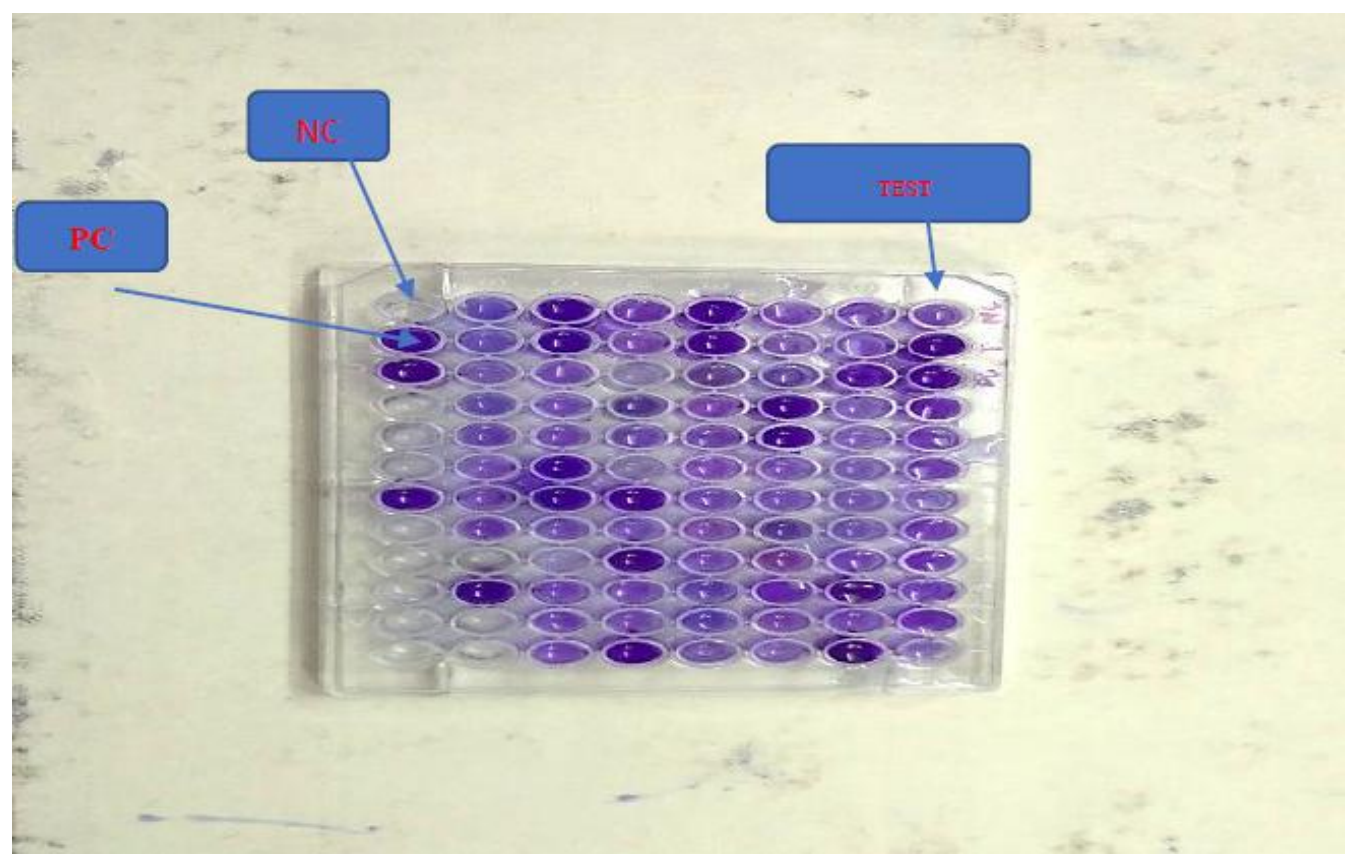

Figure.5 Biofilm production tested using microtitre plate method.

The optical density (OD) of the test, positive control and negative control is measured and biofilm production detected using the criteria elucidated in Figure 4

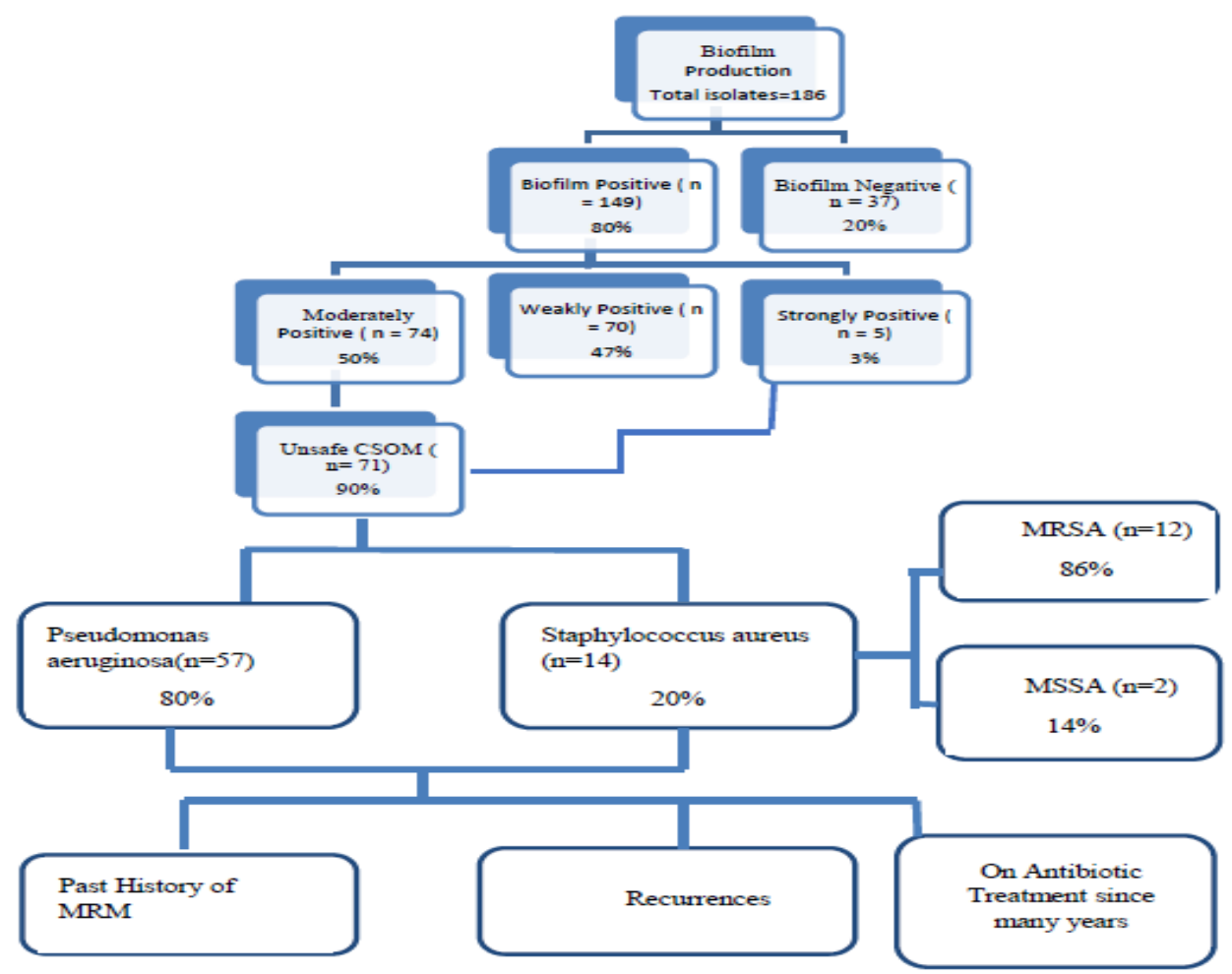

Figure.6 Flowchart showing a summary of results of Biofilm Production by Pseudomonas aeruginosa and Staphylococcus aureus 
Pseudomonas aeruginosa and Staphylococcus aureus are the most common bacterial pathogens isolated from the cases of CSOM. Biofilm production may be seen in a very high percentage of isolates and may be contribute to resistance to antibiotic, recurrent CSOM and failure of surgical procedures such as modified radical mastoidectomy. Since biofilm production appears to play a major role in pathogenesis of CSOM, it may be necessary to develop and apply in clinical practice such agents which may inhibit the production of biofilm and help in better management of CSOM.

\section{References}

Afolabi, O.A., Salaudeen, A.G., Ologe, F.E., and Nwabuisi C. (2012). Pattern of bacterial isolates in middle ear discharge of patients with CSOM. African Health Sciences 7(2):1-8.

Artono, A., Purnami, N., and Rahmawati, R. (2015) Biofilm Bacteria Plays a Role in CSOM Pathogenesis and Unsafe Type CSOM. Folia Medica Indonesiana 51(4):208-213.

Becker, K., Heilmann, C., and Peters, G. (2014)

Coagulase-Negative

Staphylococci. Clin Microbiol Rev 27(4):870 - 926.

Berman, S. (1995). Otitis media in developing countries. Pediatrics 96:126. PMID: 7596700

Costerton, J.W., Stewart, P.S., and Greenberg, E.P. (1999). Bacterial biofilms: a common cause of persistent infections. Science 284:1318-1322.

Donelli, G., and Vuotto, C. (2014) Biofilmbased infections in long-term care facilities. Future Microbiol 9(2):175-88

Hank, M.L. and Kielian, T. (2012). Deciphering mechanisms of staphylococcal biofilm evasion of host immunity. Front Cell Infect Microbiol 2(62):1-12.
Kumar, R., Agarwal, R.R., and Gupta, S.A. (2015). A Microbiological study of CSOM. IJRSR 6(7):5487-90.

Kuchal, V. (2010). Antibiotic sensitivity pattern in chronic suppurative otitis media (Tubotympani type) in Kumoun region. Indian J 16:17-21.

Kumar, S., Sharma, R., Saxena, A., Pandey, A., Gautam, P., and Taneja, V. (2012). Bacterial flora of infected unsafe CSOM. Indian J Otol 18(4):208-11.

Lee, M.R., Pawlowski, K.S., Luong, A., Furze, A.D., and Roland, P.S. (2009). Biofilm presence in humans with chronic suppurative otitis media. Otolaryngol Head Neck Surg 141(5):567-71.

Longauerova, A. (2006). Coagulase negative staphylococci and their participation in pathogenesis of human infections. Bratisl Lek Listy 107(11-12):448-452.

Li, Y.H., and Tian, X. (2012) Quorum sensing and bacterial social interactions in biofilms. Sensors (Basel) 12(3):251938.

Marchant, E.A., Boyce, G.K., Sadarangani, M., and Lavoie, P.M. (2013). Neonatal Sepsis due to Coagulase-Negative Staphylococci. Clin Dev Immunol 2013:586076.

Naheed, P., Khan, Z., Khan, I.M., Suhail, A.S., and Zuber, A. (2003). Bacteriological study of patients with discharging otitis media-A rural study.Indian J Otol 9:29-32.

Otto, M. (2013) Coagulase-negative staphylococci as reservoirs of genes facilitating MRSA infection. Nat Inst Health 35(1):4-11.

Prakash, R., Juyal, D., Negi, V., Pal, S., Adekahandi, S., Sharma, M., and Sharma, N. (2013) Microbiology of chronic suppurative otitis media in a tertiary care setup of Uttarakhand state. India N Am J MedSci 5(4)282-7.

Rabin, N., Zheng, Y., Opoku-Temeng, C., 
Du, Y., Bonsu, E., and Sintim, H.O. (2015). Agents that inhibit bacterial biofilm formation. Future Med Chem 7(5):647-71. doi: 10.4155/fmc.15.7.

Saini, S., Gupta, N., Aparna, S., and Sachdeva, O.P. (2005). Bacteriological study of paediatric and adult chronic suppurative otitis media. Indian J pathol Microbiology 48:413-16.

Schommer, N.N., Christner, M., Hentschke, M., Ruckdeschel, K., Aepfelbacher, M., and Rohde, H. (2011). Staphylococcus epidermidis uses distinct mechanisms of biofilm formation to interfere with phagocytosis and activation of mouse macrophage-like cells 774A1. Infect Immun 79:2267-2276.

Shyamla, R., and Reddy, S.P. (2012). The study of bacteriological agents of chronic suppurative otitis mediaaerobic culture and evaluation. J MicrobiolBiotechnol Res 2:152-62.

Stewart, P.S., and Costerton, J.W. (2001). Antibiotic resistance of bacteria in biofilms. Lancet 358(9276):135-138.

Wang, J.C., Hamood, A.N., Saadeh, C., Cunningham, M.J., Yim, M.T., and Cordero, J.(2014) Strategies to prevent biofilm-based tympanostomy tube infections. Int $J$ Pediatr Otorhinolaryngol 78(9):1433-8.

Wario, B.A., and Ibe, S.N. (2006).Bacteriology of chronic discharging ears in Port Harcourt, Nigerai. West Afr J Med 25:219-22.

World Health Organization . (2004). Chronic Suppurative Otitis Media. Burden of Illness and Management Options. Geneva: World Health Organization; 2004. [Accessed April 23, 2020]. Available from: http://www.who.int/pbd/deafness/activit ies/hearing_care/otitis_media.pdf.

Yeo, S.G., Par, D.C., Hong, S.M., Cha, C.L. and Kim, M.G. (2007). Bacteriology of chronic suppurative otitis media - a multicentre study. Acta Otolaryngol 127(10):1062-7.

\section{How to cite this article:}

Priya Bhat, Sameer Peer and Raksha Yoganand. 2020. Detection of Bio-film Production among the Most Frequent Bacterial Isolates in Cases of Chronic Suppurative Otitis Media: A Crosssectional Study. Int.J.Curr.Microbiol.App.Sci. 9(06): 1346-1357.

doi: https://doi.org/10.20546/ijcmas.2020.906.167 